\title{
Classe, conscience et culture : La dialectique du sujet-objet et la problématique de la réception dans I'œuvre de Lucien Goldmann
}

\author{
Ar. Gör. Dr. Utku Uraz AYDIN \\ marmara üniversitesi iletişim fakültesi gazetecilik bölümü \\ uraz.aydin@gmail.com
}

\begin{abstract}
Class, Conscience and Culture: The Dialectics of Subject-Object and the Problematic of Reception in Lucien Goldmann's Works

After the Second World War in a climate where structuralism and existentialism were the dominant currants, the cultural sociologist Lucien Goldmann, formed a dialectical methodology based on Hegel's, Marx's and Lukàcs' theoretical heritage. Genetic structuralist method is based on the category of totality, the notion of potential consciousness and the assumption of the partial identity of the subject and the object of knowledge. By applying this method on the works of writers such as Pascal, Racine, Malraux or Genet, Goldmann aims to clarify the relations between trans-individual subjects' world-view and cultural creativity. Besides, even though it is fragmented, Goldmann has a reflexion on reception problematic. Our study is an attempt to systematize the interrogations and assumptions concerning this issue. The emphasis on the human character of communication, the social determination of possibilities of reception, the central role of reification process settle on the problematic of praxis which articulated the search for the possibilities of the knowledge of the reality and the quest for its transformation.
\end{abstract}

keywords: Lucien Goldmann, genetic structuralism, reception, potential consciousness, reification 


\section{Résumé}

Dans le climat intellectuel et culturel de l'après deuxième guerre mondiale dominé par le structuralisme et l'existentialisme, le sociologue de la culture Lucien Goldmann, s'appuyant sur l'héritage théorique de Hegel, Marx et Lukacs développe une méthodologie dialectique fondée sur la catégorie de la totalité, le concept de conscience possible et le postulat d'une identité partielle entre le sujet et l'objet de la connaissance. A travers l'application de la méthode structuraliste génétique aux œuvres d'auteurs comme Pascal, Racine, Malraux ou encore Genet, Goldmann s'efforce de dévoiler les rapports entre la vision du monde de sujets transidividuels et la création culturelle. Toutefois se trouve aussi chez Goldmann une réflexion fragmentaire sur la question de la réception. Notre étude constitue une tentative de systématisation des interrogations et des hypothèses soulevées par Goldmann. L'insistance sur le caractère humain de la communication, la détermination sociale des possibilités de réception, le rôle central du procès de réification constituent les principaux points d'attaches de cette réflexion qui s'insère dans la problématique de la praxis où s'articulent la recherche des possibilités de la connaissance et la quête de la transformation de la réalité.

mots-clès: Lucien Goldmann, structuralisme génétique, réception, conscience possible, réification

\section{Öz}

\section{Sınıf, Bilinç ve Kültür: Lucien Goldmann'ın Çalışmalarında Özne- Nesne Diyalektiği ve Alımlama Sorunsalı}

İinci Dünya Savaşı sonrasında yapısalcılığın ve varoluşçuluğun hâkim olduğu entelektüel ve kültürel iklimde, kültür sosyoloğu Lucien Goldmann Hegel'in, Marx'ın ve Lukacs'ın kuramsal mirasına dayanarak özgün bir diyalektik metodoloji oluşturur. Oluşumsal yapısalcı yöntem totalite kategorisi, muhtemel bilinç kavramı ve bilginin öznesi ve nesnesinin kısmî özdeşliği varsayımına dayanır. Goldmann bu yöntemi Pascal, Racine, Malraux veya Genet gibi yazarların eserlerine uygulayarak birey-ötesi öznelerin dünya görüşleriyle kültürel yaratım arasındaki ilişkilere açıklık getirmeyi amaçlar. Bunun yanında Goldmann'da dağınık ve parçalı olmakla birlikte alımlama meselesine de ilişkin bir bakış vardır. Çalışmamız Goldmann'ın bu konudaki sorgulamalarını ve varsayımlarını bir sistematikleștirme denemesidir. Iletişimin insanal karakteri hakkındaki vurgunun, alımlama olanaklarının toplumsal belirleniminin ve şeyleşme sürecinin merkezî rolünün temel dayanaklarını teşkil ettiği bu bakış, gerçekliğin bilinmesi imkanlarıyla onu dönüştürme arayışının eklemlendiği bir praksis problematiğine oturur.

anahtar kelimeler: Lucien Goldmann, oluşumsal yapısalcılık, alımlama, muhtemel bilinç, şeyleşme 


\section{Introduction}

La position occupée par Lucien Goldmann au sein du champ intellectuel francophone de l'après-guerre, et précisément à l'intérieur des sciences sociales marxiennes, semble quasi-unique. La redécouverte de la méthode dialectique d'inspiration hégélo-marxiste à travers l'œuvre théorique du jeune Lukacs et son application dans les sciences humaines et spécifiquement dans la sociologie de la culture par Lucien Goldmann, a ouvert la voie à une revitalisation de la pensée marxiste dominée jusqu'alors par le dogmatisme stalinien. La mobilisation du principe méthodologique de totalité, d'une conception dialectique des rapports sujet-objet, des concepts de vision du monde, de médiation, de réification, de conscience possible a permis d'ouvrir une brèche dans le carcan étouffant d'un déterminisme mécaniciste disséminé par le matérialisme vulgaire d'origine soviétique.

Les travaux de Goldmann s'insèrent dans un vaste champ de recherche qui comprend des disciplines aussi variées que l'épistémologie, la philosophie politique, la psychologie et bien sûr, la sociologie de la culture. Lucien Goldmann se sert ainsi des différentes catégories et notions empruntées (en les soumettant à une reconsidération critique) à ces différents registres pour les enrichir mutuellement et les synthétiser dans le cadre de son approche structuraliste génétique. Toutefois son terrain privilégié de recherche demeure la sociologie de la culture. Pascal et Racine, Malraux et Robbe-Grillet, Saint-John Perse et Sartre, Gombrowicz et Genet sont parmi les principaux penseurs et auteurs dont l'œuvre, insérée dans la totalité socio-historique qui permis sa rédaction, est analysée afin d'éclaircir les rapports entre la création culturelle et les structures mentales des "sujets transindividuels", entre la "structure significative " de l'œuvre et la vision du monde des classes sociales.

Toutefois si ses investigations sont essentiellement centrées sur la problématique de la création, les travaux de Lucien Goldmann contiennent aussi des pistes éparses pour une réflexion sur la question de la réception. Après avoir présenté les principales lignes de la démarche méthodologique de Goldmann et donné un aperçu des catégories épistémologiques utilisées, nous essayons dans cet article de synthétiser les questionnements de l'auteur concernant les procès de communication et de réception.

\section{Notes Biographiques}

Né en 1913 à Bucarest, Lucien Goldmann grandi dans la petite ville roumaine de Botoşani. Parlant le roumain, l'allemand et le yiddish dès son plus jeune âge, Goldmann apprend le français lors de ses études secondaires. Sa politisation commence dès 1927 lorsqu'il rejoint l'organisation de jeunesse socialistesioniste Ha-Shomer ha-Tsair ("Le jeune garde »). Après son baccalauréat, il part pour Bucarest pour faire des études de droit. Lors d'un séjour d'une année 
à Vienne où il aura ses premiers contacts avec les étudiants communistes, Lucien Goldmann va suivre les cours de Max Adler, le renommé philosophe austro-marxiste. Selon son biographe Mitchell Cohen, c'est probablement sa rencontre avec Adler qui a été à l'origine de son intérêt pour Kant (auquel il consacra sa thèse de doctorat en philosophie en 1943-1944) et de la notion de transindividualité qui constituera un des piliers sur lequel sera fondé son travail théorique. II semble aussi fort possible qu'il ait rencontré lors de cette année 1930-1931 les écrits de l'Ecole de Francfort et pris connaissance des ouvrages de Georg Lukacs. Critique par rapport au stalinisme, son activité au sein du Parti Communiste Roumain de plus en plus lié au régime soviétique, ne durera que quelques années (approximativement de 1930-31 à 1935). En 1935 Goldmann s'installe à Paris pour préparer un doctorat d'économie politique à la Faculté de Droit, puis une licence d'allemand et de philosophie à la Sorbonne. Suite à l'invasion allemande en 1940, il s'enfuit à Toulouse où il est interné dans un camp dont il s'évade. Lorsque l'invasion s'étend à la Zone Française Libre, il passe clandestinement en Suisse où il est encore une fois interné dans un camp de réfugié. C'est là qu'il rencontre Manès Sperber, intellectuel communiste et disciple de l'éminent psychiatre Alfred Adler qui l'introduira (à l'intérieur même du camp) à la réflexion dialectique de Georg Lukacs exposée dans son Histoire et conscience de classe (1923). II est libéré en septembre 1943 grâce à l'intervention du Grand Rabin de Zurich et se met à rédiger sa thèse en philosophie sur Kant qui sera publié en 1945 en allemand puis en français. C'est aussi à cette période qu'il fait la connaissance du psychologue Jean Piaget dont il sera l'assistant pendant un an et dont les travaux d'épistémologie génétique (et notamment les concepts de structure mentale et d'équilibration) seront capitaux pour fonder sa propre méthodologie qu'il dénommera d'ailleurs " structuralisme génétique ". De retour à Paris après la Libération, Goldmann obtient un poste de chercheur au CNRS et se concentre sur sa thèse de doctorat ès Lettres publié en 1956 sous le titre de Le Dieu caché, étude sur la vision tragique dans les "Pensées" de Pascal et le théâtre de Racine. II publie entretemps Sciences humaines et philosophie (1952). En 1959 alors qu'il est élu directeur d'études à l'Ecole Pratique des Hautes Etudes, Goldmann publie ses Recherches dialectiques où parmi divers textes portant sur les rapports entre théorie marxiste, philosophie et sociologie de la littérature se trouve son important essai concernant la réification. Pendant les années soixante Lucien Goldmann ressent profondément l'attraction de l'expérience autogestionnaire yougoslave et sous I'impact des thèses sur la nouvelle classe ouvrière, développe sa réflexion sur le réformisme révolutionnaire qui remet en compte la centralité du prolétariat dans la lutte de classe. II assume à partir de 1964 la direction du centre de sociologie de la littérature de I'Institut de Sociologie de I'Université Libre de Bruxelles qu'il avait lui-même créé en 1961. Les recherches qu'il entreprend ici donneront lieu à la publication de Pour une sociologie du roman (1964). Deux ans avant sa mort, Lucien Goldmann aura I'occasion de participer activement aux évènements de mai 1968 aux côtés des étudiants. Ses différents essais de réflexion théorique et méthodologique seront recueillis dans des ouvrages comme Marxismes et 
sciences humaines (1970), Structures mentales et création culturelle (1970), La Création culturelle dans la société moderne (1971), Epistémologie et philosophie politique (1978) (Cohen, 1994).

\section{Totalité et Dialectique du Sujet-Objet}

"Le fait social est un fait total ( (Goldmann, 1978a, p. 47). Telle est la devise méthodologique qui guide les recherches de Lucien Goldmann. C'est donc sous le signe de la notion de totalité que se définit et s'applique la sociologie de l'auteur du Dieu caché. Sa découverte, totalement "par hasard " comme il l'affirme luimême, de l'œuvre de Georg Lukacs, fut - comme nous l'avons indiqué plus haut - essentielle pour la construction de son approche sociologique. Précisons par ailleurs que c'est l'œuvre de jeunesse du philosophe hongrois, l'Ame et les formes (1910), La théorie du roman (1916), mais principalement Histoire et conscience de classe (HCC-1923) - qui seront plus tard reniés par son auteur qui lui fournissent les principaux outils méthodologiques et épistémologiques, dont celui de totalité. Ainsi que le note Lukacs dans son $\mathrm{HCC}$ le concept est bien antérieur à sa réflexion :

Ce n'est pas la prédominance des motifs économiques dans l'explication de I'histoire qui distingue de façon décisive le marxisme de la science bourgeoise, c'est le point de vue de la totalité. La catégorie de la totalité, la domination, déterminante et dans tous les domaines, du tout sur les parties, constitue l'essence de la méthode que Marx a emprunté à Hegel et qu'il a transformée de manière originale pour en faire le fondement d'une science entièrement nouvelle (2001, p. 47).

Toutefois le mérite de Lukacs est d'avoir su réactualiser la centralité de cette notion qui disparut de la conception matérialiste de l'histoire pour toute une période au profit d'une historiosophie positiviste et progressiste-linéaire marquée par l'absence de la méthode dialectique - propagée par le marxisme officiel de la II. Internationale ${ }^{1}$. Ce point de vue de la totalité implique, toujours selon Lukacs, "la considération de tous les phénomènes partiels comme des moments du tout, du processus dialectique, qui est saisi comme unité de la pensée et de l'histoire ". Ainsi la description correcte, juste d'un évènement historique, d'un fait social n'est pas suffisante pour une compréhension de la réalité, ce n'est qu'en le saisissant dans " sa fonction réelle à l'intérieur du tout historique auquel il appartient ", à l'intérieur de "l'unité du processus

1 Martin Jay précise que "I'histoire du marxisme occidental peut être relatée en termes de I'ascension et du déclin du concept de totalité. Dans ce récit, deux des principaux protagonistes furent évidemment Georg Lukacs et Ernst Bloch, dont les rôles de pères fondateurs de la tradition marxiste occidentale ont été universellement reconnus. Non moins remarquable est l'importance que chacun d'eux - même si Bloch le fit un peu moins que Lukacs - accorda à la totalité en tant que catégorie critique d'analyse nécessaire pour sortir le marxisme de l'impasse théorique dans laquelle il était tombé pendant les quatre décennies qui suivirent la mort de Marx » (1986, p. 59). 
historique " qu'il devient possible de comprendre " la réalité en tant que devenir social » (2001, p. 31-32, 48).

De cette conception découle un autre argument essentiel de Lukacs, dans sa polémique avec d'une part la "science bourgeoise " (rationalisme moderne, empirisme, néo-kantisme etc...) et d'autre part les interprétations positivistes du marxisme. Le regard sur la réalité socio-historique ne peut être porté que de l'intérieur de la totalité. Le sujet qui tente de parvenir à la connaissance de cet objet, en constitue lui-même une partie qui grâce à sa réflexion théorique et son activité pratique (" praxis ") se transforme en même temps qu'il déstructure et restructure cet objet que constitue la totalité. Une connaissance "neutre " telle que celle fournie par les sciences naturelles n'est donc pas accessible dans le domaine des sciences humaines en raison de "l'action réciproque ", de l'existence " d'une relation dialectique du sujet et de l'objet dans le processus de I'histoire " (2001, p. 20). Lukacs réfute ainsi toute dualité, toute séparation entre le sujet et l'objet de la connaissance et défend la thèse d'une identité entre ceux-ci, à la suite de Hegel. Une présentation détaillée du travail de Lukacs dépassant le cadre de cet article, contentons-nous de souligner ces quelques points pour passer à l'interprétation qu'en fait Lucien Goldmann.

Cette " véritable encyclopédie des sciences humaines " (1978a, p. 44) que constitue HCC a été pour Lucien Goldmann une découverte capitale, comme nous l'avons précisé plus haut. C'est à travers cet ouvrage que le sociologue roumain a renoué avec la tradition dialectique dont il a été par la suite un fervent défenseur face à la fois à une nouvelle version du positivisme que représente le structuralisme -notamment althussérien- et à l'existentialisme. Son " structuralisme génétique " est justement un effort théorique visant à analyser la genèse et les transformations des structures suite à l'action réciproque entre celle-ci et les sujets collectifs, à savoir les classes et catégories sociales. II est donc question d'un structuralisme dialectique contrairement au structuralisme positiviste et althussérien faisant fi de la notion de sujet conscient et de l'histoire.

La catégorie de totalité est au centre de sa démarche, dès son travail sur Kant dont il interprète la philosophie sous l'angle de deux totalités, la communauté humaine et l'univers (1948). De même, le premier chapitre de la première partie de son opus magnum, Le Dieu caché porte sur "Le Tout et les parties ". Dans cette présentation limpide de sa méthodologie, Goldmann explique que le principe fondamental de la pensée dialectique réside dans le fait que la " connaissance des faits empiriques reste abstraite et superficielle, tant qu'elle n'a pas été concrétisé par son intégration à l'ensemble qui seule permet de dépasser le phénomène partiel et abstrait pour arriver à son essence concrète, et implicitement à sa signification » (1976, p. 17).

Pour Goldmann, la catégorie de totalité à laquelle se réfère Lukacs dans son $H C C$, tout autant que la notion de forme qui lui est apparentée et 
sur laquelle il se base dans l'Ame et les formes sont aussi exprimables sous le concept de structure dynamique significative (2001, p. 157). Ainsi dans la sociologie goldmannienne, à l'instar de l'épistémologie de Piaget, la structure est identifiée à la totalité ${ }^{2}$. Si Lucien Goldmann ne réfute pas I'horizon d'une totalité socio-historique, il n'hésite pas à forger le concept de "totalité relative" pour parler des différentes structures significatives ou structures cohérentes créées par l'activité humaines. Il est ainsi question de plusieurs niveaux de totalités plus ou moins vastes, comprenant chacune des totalités/structures en son sein, et faisant elles-mêmes parties d'autres totalités relatives, en perpétuel procès de structuration et déstructuration, donc dynamique. Car une des idées fondamentale du structuralisme génétique (et de toute sociologie dialectique, dit Goldmann) est que tout comportement humain, individuel ou collectif, a un caractère significatif, c'est à dire qu'il représente un " essai de résoudre un problème pratique ", constitue une " tentative de modifier une situation donnée dans un sens favorable à ses aspiration " (1970, p. 56; 1978a, p. 151). C'est cette conception dynamique, dialectique de la structure et le rôle déterminant attribué au facteur subjectif qui positionne le structuralisme génétique dans une opposition radicale avec le structuralisme d'un Lévi-Strauss, d'un Lacan ou d'un Barthes ${ }^{3}$. Ainsi une totalité n'est jamais "donnée ", il ne s'agit pas de structures figées mais en évolution permanente. Et c'est en raison de ce caractère dynamique de la structure, mais aussi du fait que le chercheur se trouve à l'intérieur de ces totalités qu'il devient impossible d'en parler " à l'indicatif » (1966, p. 266):

Nous essayons toujours par notre comportement et notre action de transformer ces totalités, d'avancer dans le devenir de l'histoire, de saisir de manière aussi précise que possible les réalités en les insérant dans des totalités relatives de plus en plus vastes, mais bien entendu nous n'arrivons jamais à l'objectivité. (1966, p. 267)

\section{Objectivité et Sujet de la Connaissance}

Mais est-ce que ces propos signifient que Goldmann refuse toute possibilité d'accéder à une connaissance objective dans le cadre des sciences humaines, que toute analyse est inévitablement marquée par un relativisme? $\mathrm{Ce}$

2 La définition de la notion de structure que fait Jean Piaget dans son Etude d'épistémologie génétique est reprise par Goldmann : "Nous disons... qu'il y a structure (sous son aspect le plus général) quand les éléments sont réunis en une totalité présentant certaines propriétés en tant que totalité et quand les propriétés des éléments dépendent entièrement ou partiellement de ces caractères de la totalité " $(1970$, p. 220)

3 C'est cette historicité, dialectiquement conçue, que souligne Goldmann lorsqu'il écrit que: " Tout fait humain a un caractère historique et doit être étudié comme élément ou secteur d'un processus résultant du comportement d'un ou de plusieurs sujets transindividuels, processus qui présente deux faces complémentaires: déstructuration des structures pré-existantes et structuration orienté vers la création d'un équilibre nouveau, d'une structure significative nouvelle qui sera elle-même ultérieurement modifiée et dépassée à son tour. "(1978a, p. 156157) 
n'est certainement pas le cas. On pourrait plutôt avancer, avec S. Naïr et M. Löwy que selon Goldmann il est question de deux types d'objectivité radicalement différents dans les sciences de la nature et dans les sciences sociales (1973, p. 26). La différence fondamentale entre les deux est que la première étudie "les faits uniquement sur le plan extérieur de leur réalité sensible " alors que la deuxième a pour but d'analyser des actions accomplies consciemment, dont il doit prioritairement rechercher la signification (1978a, p. 29). Mais d'autre part, comme nous l'avons indiqué plus haut, le travail pour accéder à une connaissance scientifique est lui-même le résultat d'actions humaines, historiques et sociales. Ceci implique donc, pour Goldmann, " l'identité partielle entre le sujet et l'objet de la connaissance » (1978a, p. 33). Nous le remarquerons tout de suite, Goldmann opère ici une atténuation du caractère absolu de la prémisse épistémologique de Hegel/Lukacs. C'est le deuxième remaniement des principaux termes de la dialectique lucaksienne, après la construction de la notion de totalité relative. Cela signifie que le sujet de la réflexion (ou de la recherche, de l'analyse...) et le processus de connaissance sont des aspects sans doute très importants de la réalité, mais ne constituent que des aspects et non la totalité de la réalité (1970, p. 55). De plus, la nature de cette identité partielle est variable dans chaque cas particulier et doit être, selon Goldmann, précisée au moment où le chercheur aborde l'étude de ce cas spécifique (2001, p. 187). Donc la question de l'objectivité se pose à un niveau autre que dans le modèle scientifico-naturel, qui au fil des siècles, a réussi à atteindre un niveau de scientificité libre de préjugés idéologiques et de jugement de valeurs. Ou du moins le principal jugement de valeur est partagé par l'ensemble des différentes classes et catégories sociales : la domination de la nature. ${ }^{4}$

Pour en revenir à la question de l'objectivité dans les sciences humaines, Lucien Goldmann, contrairement à Emile Durkheim, ne pense pas qu'il soit possible d'étudier les faits sociaux "comme des choses ". Pour lui ce n'est pas une question relevant de l'immaturité de la sociologie ou bien d'un manque d'objectivité dû aux défauts personnels du chercheur. C'est une impossibilité épistémologique, que la force de pénétration ou bien l'honnêteté intellectuelle de chercheur ne saurait combler. Car ce dernier qui occupe une certaine position dans la société et dont les structures mentales ont été forgées dans un certain processus historique, " aborde le plus souvent les faits avec des catégories et des prénotions implicites et non conscientes qui lui ferment d'avance le chemin d'une compréhension objective ". Goldmann s'oppose aussi à l'idée de Max Weber qui, ne rejetant pas le poids des jugements de valeur, affirme qu'il serait possible de limiter l'intervention de ces jugements seulement dans le choix et la construction de l'objet, et de mener par la suite une étude de manière objective. II souligne le caractère erroné de cette idée : "Les éléments choisis déterminent d'avance, cela va de soi, le résultat de l'étude. Les valeurs étant non les 'nôtres',

4 Toutefois, soulignons au passage, qu'avec la dégradation de l'équilibre écologique, les sciences naturelles, avec par exemple l'interprétation des rapports sur le réchauffement de la planète sont redevenus un champ de combat idéologique et politique. 
celles de notre culture ou de notre société, mais celles de telle ou telle classe sociale, ce qu'une perspective éliminera comme non essentiel, peut-être, au contraire, très important dans une autre " (1978a, p. 33-45).

Mais alors quelle serait la possibilité d'atteindre une connaissance adéquate de la réalité sociale ? A l'instar de Georg Lukacs, Goldmann défend l'idée qu'au niveau collectif, transindividuel, au long du processus historique certaines classes sociales en ascension acquièrent la capacité d'accéder à une connaissance plus objective, moins déformante de la totalité socio-historique. Dans une société basée sur les rapports de production capitaliste, c'est la classe ouvrière qui posséderait cette supériorité épistémologique. Comme classe qui tend à l'abolition de l'oppression, à la suppression de l'existence même des classes, elle s'identifie ainsi avec l'Humanité. Selon Goldmann,

\begin{abstract}
Il [le prolétariat] est à la longue la première classe qui tend vers une connaissance vraie et sans réserve aussi bien du monde physique que du monde sociale. C'est pourquoi il a une attitude positive devant tout résultat scientifique qui augmente notre connaissance de la réalité et une attitude entièrement négative envers toutes les idéologies qui nient en totalité ou en partie la valeur ou l'importance de la science (1980, p. 22-23).
\end{abstract}

N'ayant donc aucun intérêt à dissimuler des zones particulières de la réalité humaine et du fait de sa position dans les processus de production, elle est, par rapport aux classes en déclin, plus apte à soulever le voile des procès de réification et à rendre compte de la complexité des rapports sociaux. C'est dans le cadre de cette épistémologie liée à la conscience de classe (où, selon Lukacs, la conscience et la science tendent à se confondre) qu'entrent en jeu les concepts de vision du monde, conscience possible et structures mentales.

\title{
Création Imaginaire et Vision du Monde
}

La distinction primordiale à opérer, lorsqu'il est question d'analyser des faits de conscience collective et plus spécifiquement le niveau d'adéquation ou d'inadéquation à la réalité de la conscience des différents groupes sociaux, doit être selon Goldmann, celle entre la conscience réelle et la conscience possible.

La première est la conscience effective, celle que les membres d'un groupe, catégorie ou classe sociale possèdent à un moment donné de l'évolution historique et dans des conditions externes précises. C'est celle que les partisans des méthodes positivistes, purement descriptives reconnaissent comme conscience en tant que telle. Au niveau des classes sociales, elle pourrait s'apparenter à ce que Marx définit comme celle de la classe-en-soi. Cette conscience réelle que chaque classe sociale a à propos des différentes questions que la réalité - qui est elle-même formée par la conjoncture sociohistorique - lui pose, résulte des diverses limitations, obstacles, déviations 
que les multiples facteurs de la réalité empirique font subir à sa conscience de classe. Parmi ces facteurs, Goldmann cite d'une part les facteurs naturels, voir " cosmiques " et d'autre part les effets des actions des autres groupes sociaux.

Le concept de conscience possible auquel se réfère Goldmann a été emprunté, encore une fois à Lukacs. L'auteur de Histoire et conscience de classe parle en fait de " conscience adjugée » (Zugerechnetes Bewusstsein). C'est selon Goldmann la conscience " calculée " ou " construite " par le chercheur. Chez Lukacs cette notion va de pair avec celle de possibilité objective. En établissant le rapport de la conscience à la totalité concrète socio-historique, on découvre, ou bien pour atténuer un tant soit peu le caractère absolu des propos de Lukacs - on acquiert la possibilité de découvrir les pensées, sentiments, représentations que les membres d'un groupe social auraient eu dans une situation précise et déterminée, s'ils avaient détenu la capacité de saisir, de manière adéquate à la réalité, cette situation ainsi que les conséquences probables de leur action collective sur celle-ci. Ce n'est donc " ni la somme ni la moyenne de ce que les individus qui forment la classe, pris un par un pensent, ressentent (...) mais la réaction rationnelle adéquate qui doit être adjugée à une situation typique déterminée dans le processus de production " (Lukacs, 2001, p. 73). II est donc, une fois de plus, question d'une conception dialectique des rapports de détermination entre la conscience/la connaissance et la réalité concrète mais dynamique, conçue comme un champ de possibilités. La conscience possible est déterminée par la possibilité objective, qui elle-même est constitué par celleci. Dans son article sur l'Epistémologie de la sociologie Goldmann souligne le fait que la catégorie de conscience possible constitue ainsi une " schématisation opératoire " qui permet au chercheur de connaître le maximum de conscience possible que peut atteindre un groupe social, c'est-à-dire le champ à l'intérieur duquel les connaissances et les réponses à la situation donnée peuvent varier sans qu'il y ait une modification essentielle dans sa structure (1978b, p. 34-35).

L'utilisation de la notion de vision du monde a pour objectif de formuler une typologie des consciences possibles, des maximums de conscience que les structures mentales des classes sociales peuvent atteindre. II n'est donc pas question d'une donnée immédiate, empiriquement observable mais d'un instrument conceptuel de travail. Traduction française de la Weltanschauung telle qu'elle fut formulée par Wilhelm Dilthey, la vision du monde exprime " une vision d'ensemble de l'homme actuel, de ses qualités, de ses défauts et un idéal de l'humanité future, de ce que doivent être les relations de l'homme avec les autres hommes et avec l'univers ". Toutefois une vision du monde cohérente ne peut être construite par tous les groupes sociaux. Les intérêts collectifs de ces groupes doivent être orientés soit vers une transformation d'ensemble, soit vers un maintien global de la structure sociale contemporaine dans laquelle ils œuvrent. C'est donc " précisément cet ensemble d'aspirations, de sentiments 
et d'idées qui réunit les membres d'un groupe (le plus souvent, d'une classe sociale) et les oppose aux autres groupes » $\left(1976\right.$, p. 26) ${ }^{5}$.

Nous ne pouvons pas parler de l'élaboration systématique d'une typologie des différentes visions du monde correspondant au maximum de conscience possible de diverses classes sociales chez Goldmann. Mais il approfondit son étude de certaines visions du monde dans la mesure où elles permettent un développement de l'analyse structuraliste génétique d'œuvres philosophiques et littéraires. Le nombre de visions du monde est limité, et les principales Weltanschauung modernes cités et étudiés (inégalement) par Goldmann sont l'individualisme (rationalisme, empirisme), la vision tragique, le romantisme, l'existentialisme, la pensée dialectique ${ }^{6}$.

L'utilisation du concept de vision du monde est particulièrement féconde au niveau de la sociologie de la culture et principalement de celle des créations imaginaires (littéraires, théâtrales et même plastiques - comme par exemple l'étude de Chagall par Goldmann). Car pour une analyse structuraliste génétique, ainsi que le souligne l'éminent germanologue Jean-Michel Palmier, ce n'est pas la forme expressive, mais la vision du monde qui sous-tend la création esthétique qui est l'objet de l'étude (1974, p. 166). Car dans la sociologie goldmannienne chaque grande et authentique œuvre littéraire ou philosophique est l'expression d'une vision du monde, "d'un point de vue cohérent et unitaire sur l'ensemble de la réalité ", "d'une manière de voir et de sentir un univers concret d'êtres et de choses ${ }^{7}$. Autrement dit, c'est dans le mesure où une œuvre réussit à

5 Disciple " néo-goldmannien de gauche » (selon sa propre expression) de l'auteur du Dieu Caché, Michael Löwy dans son remarquable ouvrage sur la sociologie critique de la connaissance (Paysage de la Vérité, Paris: Anthropos, 1985; republié sous le titre Les aventures de Karl Marx contre le baron de Münchausen, Paris: Syllepse, 2012) définit la vision sociale du monde comme " un ensemble organique, articulé et structuré de valeurs, représentations, idées et orientations cognitives, intérieurement unifié par une perspective déterminée par un certain point de vie socialement conditionné ". (1985, p. 13)

6 Pour une étude sur la vision romantique voir M. Löwy-R. Sayre, Révolte et mélancolie. Le romantisme à contre-courant de la modernité (Paris: Payot, 1992). Pour un essai d'analyse structuraliste génétique de l'œuvre de J.R.R. Tolkien dans le cadre de la vision romantiqueanticapitaliste voir. U. Uraz Aydin, Sihir ve Ütopya. Tolkien'in Yüzüklerin Efendisi'nde Romantik Eleştiri (Magie et utopie. La critique romantique dans le Seigneur des anneaux de Tolkien), Istanbul: Versus, 2008.

7 Son débat lors du Deuxième colloque international sur la sociologie de la littérature (Royaumont, 12-14 janvier 1968) avec Theodor W. Adorno à ce sujet est fort intéressant. Adorno ne partage pas l'avis de Goldmann sur le fait qu'une œuvre authentique doit exprimer de façon cohérente la vision du monde d'un groupe social: "Ce n'est nullement la valeur des œuvres d'art dont l'unité dans la multiplicité est la plus réussie qui est automatiquement la plus haute. II y a des œuvres d'art qui, précisément par leur caractère fragmentaire - et je considère le fragment comme une forme - s'élèvent au-dessus de cette unité systématique et qui ont des qualités qui dépassent I'unité ». Face à cette critique Goldmann réplique que le fragment peut lui aussi être un élément d'une expression cohérente: "Pour le fragment, j'admets qu'une œuvre est valable très souvent lorsqu'elle est fragmentaire - j'ai analysé moi-même deux fois des fragments, les Pensées de Pascal et des fragments de Valéry -, mais dans chaque cas il s'agit de fixer ce caractère 
exprimer de façon cohérente une vision du monde, qu'elle peut être désignée comme philosophiquement ou esthétiquement valable. Toutefois comme nous l'avons vu, les visions du monde ne sont pas des faits individuels mais sociaux, en rapport avec les structures mentales correspondant au maximum de conscience possible de certains groupes sociaux. Ainsi la grande majorité des individus de ce groupe-cette classe ne réalise ce système de pensée que partiellement et non intégralement mais au total de façon suffisante pour constituer une communauté de sentiments, de pensée et d'action qui les rapproche. Les artistes, philosophes, écrivains, par contre, " pensent ou sentent cette vision jusqu'à ses dernières conséquences et l'expriment à travers le langage, sur le plan conceptuel ou sensible " (1980, p. 46-49). Ainsi l'analyse de l'œuvre doit s'efforcer de mettre en lumière la cohérence interne et structurale, la " structure significative " qui réside en son sein, en séparant l'essentiel de ce qui est secondaire et accidentel. 8

C'est la vision tragique qui est étudiée de façon la plus approfondie par Goldmann, à travers I'œuvre de Pascal et Racine dans son ouvrage Le dieu caché. Le processus d'analyse appliqué ici, et qui constitue une des principales spécificités de la méthodologie goldmannienne présente deux principaux aspects. Le premier palier est ce qu'il appelle la compréhension, qui " est la mise en lumière d'une structure significative immanente à l'objet étudié, dans le cas précis à telle ou telle œuvre littéraire " $(1970$, p. 66.) Le second aspect, l'explication, résulte de l'insertion de cette première structure (le texte) dans la structure immédiatement englobante. Cette dernière n'est à ce niveau analysée de façon non détaillée, mais juste dans la mesure où il est nécessaire pour permettre de comprendre la genèse de l'œuvre. Mais par la suite c'est la structure englobante qui est prise comme objet d'étude et est soumise à l'analyse compréhensive et doit être expliquée par l'exploration d'une structure encore plus vaste 9 .

fragmentaire dans la totalité de l'œuvre qui se traduit en système. II n'y a pas d'opposition entre système et fragment, un fragment peut être un élément de la systématisation dans la traduction philosophique " (Adorno-Goldmann, 1973, p. 17, 19)

8 Cette citation tirée de son article sur Le concept de structure significative dans I'histoire de la culture, nous montre bien toute la charge dialectique que contient la méthodologie du sociologue roumain : "Les œuvres valables (...) se caractérisent en effet par l'existence d'une cohérence interne, d'un ensemble de relations nécessaires entre les différents éléments qui les constituent et, chez les plus importantes d'entre elles, entre le contenu et la forme, de sorte qu'il est non seulement impossible d'étudier de manière valable certains éléments de l'œuvre en dehors de l'ensemble dont ils font partie et qui seul détermine leur nature et leur signification objective, mais aussi que la possibilité de rendre compte de la nécessité de chaque élément par rapport à la structure significative globale constitue le guide le plus sûr du chercheur. " (1980, p. 107)

9 Lucien Goldmann éclaircit dans ce passage fréquemment cité, les différents niveaux d'analyse de sa méthodologie en prenant pour exemple son étude sur la vision tragique : "Comprendre Les Pensées ou les tragédies de Racine, c'est mettre en lumière la vision tragique qui constitue la structure significative régissant l'ensemble de chacune de ces œuvres; mais comprendre la structure du jansénisme extrémiste, c'est expliquer la genèse des Pensées et les tragédies raciniennes. De même, comprendre le jansénisme, c'est expliquer la genèse du jansénisme 
Bien entendu l'analyse de ces divers niveaux, ces différents découpages d'objet, de structures significatives ne sont pas réalisés de façon mécanique, il s'agit d'un va et vient permanent, dans la mesure où c'est nécessaire pour éclaircir la genèse de l'œuvre.

\section{La Dialectique de la Réception sous I’Angle de la Praxis Humaine}

La réflexion développée par Lucien Goldmann sur les questions relevant directement des problèmes de la communication et de la réception n'atteint bien sûr pas le même niveau de systématisation que l'approche structuraliste génétique dans le domaine de la création culturelle. Ce sont plutôt des réflexions fragmentaires développées à l'occasion de colloques et séminaires concernant la communication et les médias. Mais tous ses questionnements émanent bien entendu de ses études antérieures sur la sociologie de la culture et des notions, approches et catégories qui y furent développées et employées.

La réflexion de Goldmann sur les procès de communication-réception proprement dite est spécifiquement développée dans deux interventions. La première intitulée "L'importance du concept de conscience possible pour la communication " a été faite au colloque sur "Le concept d'information dans la science contemporaine ", organisé par le Centre culturel international de Royaumont en collaboration avec l'Association internationale de Cybernétique en 1962. Parmi les divers contributeurs composés de mathématiciens, physiciens, biologistes et philosophes, participèrent notamment à ce colloque Norbert Wiener, fondateur des sciences de la cybernétique, I'historien des sciences Giorgio de Santillana, le mathématicien Benoit Mandelbrot, les philosophes Jean Hyppolite et Maurice de Gandillac, I'anthropologue Lucien Sebag.

La seconde est une communication portant le titre de "Possibilités d'action culturelle à travers les mass-médias " (envoyée depuis Chicago) au séminaire international " Mass-média et création imaginaire " réalisé à Venise en 1967 sous le patronage de l'Institut de Sociologie de l'Art (Faculté des Lettres de Tours) et l'Association Internationale pour la liberté de la culture, avec la participation, entre autre, des sociologues Henri Lefebvre et Edgar Morin et de I'écrivain George Perec ${ }^{10}$.

Les différentes interrogations soulevées par Lucien Goldmann et les hypothèses qu'il formule s'insèrent dans la problématique de la praxis où s'articulent la recherche des possibilités de la connaissance et la quête de la transformation de la réalité. Ses réflexions sont donc globalement structurées

extrémiste ; comprendre I'histoire de la noblesse de robe au XVIlème siècle, c'est expliquer la genèse du jansénisme ; comprendre les relations de classe dans la société française du XVIlème siècle, c'est expliquer l'évolution de la noblesse de robe, etc. " (1970, p. 66)

10 Les deux textes ont été repris dans l'ouvrage La création culturelle dans le monde moderne (1975, Paris: Denoël/Gonthier). 
par une conception dialectique des rapports sujet-objet, dont nous avons donné un aperçu plus haut.

Parmi les divers points que Goldmann souligne et développe de façon plus ou moins approfondie, le premier est le rappel du caractère humain de la communication et à plus forte raison de la réception. Ce constat redouble d'importance dans la mesure où le colloque avait pour objectif une confrontation ou disons un échange de point de vue entre des chercheurs venant des sciences " exactes " et des philosophes et chercheurs en sciences sociales. Donc face à des conceptions techniques, cybernétiques et mathématiques des procès de " transmission d'information " (en nombre de bits par exemple) Goldmann souligne le facteur humain, en dehors de toute la "chaîne d'appareils et de machine " à travers lesquels circulent les messages. Cet accent mis sur I'humain et donc le sujet humain fait bien sûr écho au débat qu'il mène contre la tendance structuraliste (de laquelle se revendique d'ailleurs Lucien Sebag, présent à la réunion) dans les sciences sociales et la pensée critique en générale (1975, p. 7-9).

Le deuxième point important est que Goldmann se focalise d'emblée sur l'instance de la réception, en défendant l'idée que "en fin de compte, il y a toujours, au bout de la chaîne un être humain, et nous savons que sa conscience ne peut pas "laisser passer" n'importe quoi n'importe comment " (1975, p. 9). La prise en compte de la problématique de la réception constitue un des moments charnières des études sur la communication. Le paradigme centré davantage sur les effets que suscitent ou peuvent susciter les médias sur le public, qui va de l'attribution (en générale spéculative) d'un pouvoir de persuasion quasi-absolu (comme le modèle de la seringue hypodermique Lasswellienne ${ }^{11}$, pour ne citer que la plus connue) à la théorie des effets limités (la fameuse " two-step flow of communications "- déduites d'importantes recherches empiriques - de Paul Lazarsfeld) a dominé les recherches sur la communication des années vingt jusqu'au début de la décennie 1960. La problématique de la réception fut introduite avec l'approche des " usages et gratifications " qui se propose d'étudier/de mesurer (avec un "excès de positivisme " selon le sociologue de la communication Éric Maigret) non plus ce que les médias font aux gens, mais ce que ces derniers font avec les médias. Toutefois c'est avec le courant des cultural studies, initié par des sociologues et historiens marxistes comme E.P. Thompson, Richard Hoggart et Raymond Williams que la rupture avec le paradigme dominant sera définitivement consommée : I'émetteur finira d'être le seul acteur du procès de communication et le récepteur se libérera da sa position de passivité. L'article de Stuart Hall sur le " codage-décodage " (1973) des contenus-messages médiatiques sera une première théorisation des différentes modalités de réception-décodage, qui demeure toujours être

11 Selon ce modèle, les médias " injectent " les informations dans l'esprit des personnes et en les influençant directement déterminent ainsi leurs visions des choses. 
une référence incontournable sur la question (Maigret, 2004; Derville, 1997; Mattelart-Neveu, 2008).

C'est donc la structure de cette " conscience réceptrice " que se propose d'étudier Lucien Goldmann. Le processus de réception peut se conclure de trois principales façons selon lui. L'information peut bien entendu être intégralement reçue ; la conscience du récepteur, du fait de sa structure, peut-être opaque à toute une série d'informations et ne pas les "laisser passer "; ou bien elles peuvent passer mais de manière déformée, c'est-à-dire qu'une partie de l'émission est reçue et que " même cette partie a pris au niveau de la réception, une signification assez différente de celle qui avait été envoyée " (1975, p. 9). Nous devons reconnaître que les trois modes de réception énumérés schématiquement par Goldmann possèdent une certaine affinité avec celles que Stuart Hall analysera dix ans plus tard - de façon certes plus systématique et développée - dans son texte pionner. En effet Hall repère trois "positions hypothétiques " à partir desquelles la réception - le décodage - des discours médiatiques peuvent être réalisées. La position dominante-hégémonique est celle où le récepteur décode le message en fonction du code de référence initial et intègre directement le sens de ce message. Dans la position négociée, le spectateur reconnaît la légitimité des définitions et du sens hégémonique dans ses grands traits, mais à un niveau plus limité, local, il pose ses propres règles de base. II est donc question d'un " mélange d'éléments adaptatifs et oppositionnels ". Et finalement la position oppositionnelle où le récepteur détourne le sens du message en le décodant de manière globalement contraire pour en reconstruire le sens dans un autre cadre de référence (Hall, 1997).

Mis-à-part ces trois possibilités selon lesquelles le processus de transmission peut se conclure, Goldmann propose différents paliers d'analyse pour étudier le problème de transmission-réception.

Le premier est celui où le message, le discours n'est pas reçu en raison du manque d'information préalable. Goldmann donne l'exemple d'une formule de mathématique complexe qui ne peut être reçue - comprise - que grâce à une série d'informations complémentaires.

Un autre qui se trouve toujours au niveau individuel relève de la structure psychique. En invoquant Freud, Goldmann rappelle l'existence d'éléments structuraux de désir et de répugnance résultant de la biographie de l'individu qui rend son moi conscient " imperméable à certaines informations " alors qu'il attribue un sens déformé à d'autres. Ce n'est que par une transformation de la structure psychique de l'individu et non par un changement social que le message peut être reçu.

Un troisième palier, d'ordre sociologique cette fois concerne les cas ou des groupes sociaux, du fait de la structure de leur conscience réelle, qui peut 
provenir de son vécu, de différentes expériences antérieures, peut résister au passage de certaines informations. Goldmann cite l'exemple imaginaire d'un groupe de chercheur appartenant à un certain courant scientifique, qui refuse d'assimiler une nouvelle théorie susceptible de remettre en cause les thèses auxquelles il est attaché.

Enfin le quatrième niveau est en rapport avec les limites de la conscience possible. Dans ce cas-là, la transmission de certaines informations est incompatible avec les caractéristiques fondamentales de certains groupes sociaux, c'est-à-dire qu'elles dépassent le maximum de conscience possible de ces groupes (1975, p. 11-14). Ainsi devient-il important d'analyser les structures mentales du groupe social en question:

\begin{abstract}
Quelles sont les catégories intellectuelles fondamentales, l'aspect spécifique des concepts d'espace, de temps, de bien, de mal, d'histoire, de causalité, etc. qui structurent sa conscience, dans quelles mesures ces catégories sont liées à son existence, quelles sont les limites du champ de conscience qu'elles engendrent et enfin quelles sont les informations situées au-delà de ces limites et qui ne peuvent plus être reçues sans transformation sociale fondamentale. " $(1975$, p. 14)
\end{abstract}

Pour imager ses propos, Lucien Goldmann cite un exemple d'analyse de la conscience possible en vue d'une transmission d'information, pour le moins étonnant. En effet, il est question de Lénine et de la révolution d'octobre... Pour obtenir le soutien de la paysannerie qui dans sa majorité était fidèle au Tsar, Lénine " qui sur ce point faisait œuvre de théoricien de l'information ", proposait de réviser, du moins tactiquement, le principe d'opposition des socialistes à la propriété individuelle de la terre. Car il était selon lui impossible de leur faire accepter qu'il serait plus avantageux de travailler en coopérative dans de grandes exploitations que de posséder leur propre morceaux de terre. Ceci dépasserait le maximum de conscience possible de la paysannerie russe du début du siècle. Ainsi malgré l'opposition de bon nombre de socialistes, Lénine formula le nouveau mot d'ordre de "la terre aux paysans " qui permis de rallier une partie de la paysannerie au mouvement révolutionnaire (1975, p. 9-10).

Ici, nous le voyons clairement, Goldmann tente d'esquisser, de façon bien entendu parcellaire, une notion de détermination sociale des possibilités de réception. Cette question est d'ailleurs abordée dans divers textes de Goldmann, dans un autre registre, à propos de "l'influence ". Le fait qu'un tel penseur soit influencé par un autre (par exemple Althusser par Lévi-Strauss) ne constitue pas, selon Goldmann, un facteur explicatif, "c'est une donnée qu'il s'agit d'expliquer ". II faut d'abord, selon lui, se demander pourquoi un tel personnage, à un moment donné de l'histoire, de son histoire, a subit l'influence d'un tel courant et non pas d'un autre (1978b, p. 114). 
Cependant il n'est bien sûre pas question d'une détermination mécanique mais bien dialectique : La classe sociale en question ne peut recevoir, assimiler certains contenus du fait des limites de sa conscience possible - qui est, elle aussi, déterminée socialement et historiquement à travers l'accumulation de multiples expériences collectives, de ses conditions d'existences, de ses intérêts immédiats imager - mais la réception de contenus adaptés à son champs de conscience peuvent susciter une transformation de celle-ci, qui lorsqu'elle est accompagnée de l'action collective qui correspond à ce niveau de conscience, peut provoquer tout une série de transformations changeant tout à la fois les conditions d'existence et donc les caractéristiques fondamentales de cette classe, de même que les cadres cognitifs structurant sa conscience.

D'autre part, tel qu'il est possible de le constater, la notion " d'information " est considérée dans son sens plutôt large. Ce qui d'ailleurs provoque les critiques d'autres contributeurs, qui lors du débat qui suit l'intervention de Goldmann, lui répliquent qu'il a tendance à confondre information et recommandation, voire propagande. Mais pour Lucien Goldmann, informations et recommandations ne peuvent pas être conçues indépendamment les unes des autres, de même qu'il est impossible de séparer, dans les sciences humaines les jugements de fait des jugements de valeurs. II souligne d'une part l'influence des jugements de valeurs lors de l'élaboration des informations (soit disant " objectives " et conçus donc comme des jugements de fait) et affirme d'autre part que les informations en accédant à la connaissance des hommes, auront nécessairement une influence sur leur comportement et feront donc effet de recommandation. Et il ajoute finalement que lorsqu'un groupe social résiste à la réception d'une recommandation, il résistera aussi à toutes les informations susceptibles de la favoriser (Cahiers de Royaumont, 1965).

Un troisième aspect de l'approche goldmannienne sur la question de la communication et du procès de réception est le phénomène de la réification, un problème cher au sociologue roumain. Ce phénomène dont les effets sur la conscience furent auparavant analysés par Marx et Lukacs dans les sociétés capitalistes libérales puis monopolistiques s'est amplifié dans les sociétés industrielles occidentales (désignées aussi comme capitalisme d'organisation, société de consommation ou de masse). La prédomination de la valeur d'échange des marchandises sur leur valeur d'usage dans la vie sociale se reproduisant dans la conscience, cette dernière tend à appréhender les rapports sociaux entre êtres humains comme des rapports entre des choses. Ainsi le caractère global des relations interhumaines tend à disparaître de la conscience laissant sa place à une vision quantitative et abstraite au détriment des structures qualitatives, des valeurs positives, du sentiment de communauté et de l'espoir de dépassement : "La réalité perd toute transparence et devient opaque, I'homme devient limité et désorienté ; le progrès considérable des forces productives et, avec elles, de la science et de la technique, ne se réalise qu'au prix d'un énorme rétrécissement 
du champ de la conscience, surtout en ce qui concerne les possibilités de I'homme et la nature de ses relations avec ses semblables » $(1975$, p. 30)

C'est la discordance entre la quantité accrue d'information transmise par les médias et ce rétrécissement du champ de la conscience que fait ressortir Goldmann comme un des problèmes fondamentaux qui caractérisent la société moderne de consommation. C'est ce contraste qui rend compliquée la réalisation de toute tentative " d'action culturelle " émancipatrice à travers les moyens de communication. Car celle-ci pour être assimilée, nécessiterait une considérable activité de synthèse, activité que le procès de réification et ses effets sur les potentialités cognitives ont rendue particulièrement difficile. De plus, la masse d'informations émise tous les jours et en grandes quantité peut constituer un élément de désorientation et d'affaiblissement de la compréhension et d'une réception active. Cette dernière diverge d'une réception passive, qui se contente de subir le message, par le fait qu'elle cherche dans le contenu du message (information, livre, film...) " une invitation à la réflexion " en vue d'intégrer des éléments de ce contenu " dans une vision globale en la perfectionnant ou en la modifiant $"(1975$, p. 38).

Mais ainsi que le constate Lucien Goldmann, c'est la tendance à la passivité qui est en vigueur dans la société contemporaine technocratique, provoquée par une dynamique de réification accrue et précisément par l'intervention des organismes dirigeant de la production et de l'Etat dans la vie privé à travers la consommation. II est ainsi question d'un danger croissant de "déculturisation par la désorganisation des récepteurs " (1975, p. 37-38). La stratégie à adopter pour inverser cette tendance, pour régénérer l'activité culturelle et pour rendre efficace l'action culturelle à travers les médias (c'est-à-dire pour établir les bases matérielles d'une réception active) devient ainsi, dans la réflexion de Goldmann, un problème centrale. II esquisse deux " points d'impact " pouvant servir d'éléments à la formulation d'une telle stratégie. Le premier serait le développement (tout d'abord) puis, bien entendu, la réalisation, même partielle, de l'idée de démocratie économique et d'autogestion, que peut permettre selon lui, la formation de la "nouvelle classe ouvrière "12. La récupération par les travailleurs de leurs responsabilités, en se rendant actif au niveau de la décision dans le processus de production peut donc inverser cette tendance. Une autre hypothèse stratégique, réside dans la possibilité de transformation d'éléments importants de la vie quotidienne, à savoir l'augmentation du niveau de vie et du temps libre. Ceci pourrait faire surgir, du moins dans certaines couches sociales " des tendances renforcées à organiser la vie privée et à lui donner un sens, tendances qui pourraient être le point de départ d'une action de résistance à la

12 La conscience de cette nouvelle classe moyenne salariée " s'orientera très probablement vers des revendications non pas quantitatives mais qualitatives, vers une exigence de participation aux décisions et, à la limite, vers une gestion entièrement démocratique de l'entreprise ". Ainsi la perspective d'une évolution des sociétés industrielles se trouve à la fois réactualisée et profondément modifiée" (1970, p. 351-352). 
société technocratique, à condition, bien entendu, de dépasser un jour le niveau de cette vie privée et d'aboutir à une problématique globale » (1975, p. 39-40).

Toutefois Goldmann n'omet pas de souligner le risque de n'intervenir que sur le plan culturel ou à un niveau seulement politique et/ou économique. Les différents aspects de l'oppression étant devenus plus indissociables que jamais, l'action isolée sur uniquement l'un de ces plans se traduirait par une inefficacité. C'est donc une action conjointe, articulée à ces différents niveaux qui seule peut permettre de briser le cercle vicieux de la domination et de la réification (1975, p. 44-45).

\section{Conclusion}

Nous avons essayé au long de cette étude de présenter les principaux points de la sociologie goldmannienne en privilégiant ses aspects relevant de la conception dialectique - qui structure en fait toute sa méthodologie - pour ensuite analyser l'approche de l'auteur du Dieu caché concernant le procès de communication et plus particulièrement le phénomène de la réception. Tout en essayant de montrer la façon par laquelle Goldmann mobilise les notions de son structuralisme génétique pour comprendre ce processus, nous avons souligné que le fait même qu'il s'intéresse à la problématique de la réception est tout à son honneur, étant donné que ceci constitue un changement de paradigme dans la sociologie de la communication. Toutefois il faudrait préciser qu'il existe une tension entre deux conceptions de la réception chez lui. En effet, Goldmann, d'une part souligne l'importance du caractère actif que doit posséder la réception, celle-ci devant provoquer une stimulation de la conscience et aboutir à une réflexion en partant du contenu du message reçu. Mais d'autre part, lorsqu'il est question des différents paliers d'analyse du procès de réception, ce sont les possibilités de réception adéquate des messages transmis qui guident sa réflexion. La problématique est celle de pouvoir surmonter les obstacles cognitifs et la résistance à la réception du contenu. La réception est donc considérée ici du point de vue de l'émetteur, qui est pour Goldmann, celui qui « veut intervenir dans la vie sociale " (1971, p. 11). Mais cette tension entre ces deux pôles, voire la contradiction entre la réception active et la nécessité d'une transmission adéquate du message est surmontée, ou disons, aufgehoben - dans un triple mouvement de négation, conservation et dépassement dans la meilleure tradition de la dialectique hégélienne - à un niveau supérieur, qui est celui d'un impératif d'émancipation inséparablement individuelle et collective/sociale. Car comme nous le voyons explicitement dans l'exemple de Lénine et des paysans russes, la motivation principale de sa réflexion théorique, que ce soit dans ses analyses sur la réception ou bien dans ses études sociologiques sur la création culturelle, est politique, au sens large du terme. Elle est fondée sur le "pari " 
- d'origine pascalienne ${ }^{13}$ - de la possibilité d'un monde où il y aurait plus de liberté et plus de communauté humaine. Cette ambition, ce pari raisonné - qui guide tout son travail théorique -, trouve ses fondements dans la dialectique du sujet-objet, dans la " dialectique de l'immanence " (Zima, 1973) qui repose sur l'existence d'un sujet transindividuel immanent, d'une force critique interne à la réalité sociale, capable de la concevoir dans sa totalité et de la transformer, la " dépasser ". C'est cette " foi " en la praxis humaine et collective - nécessaire mais incertaine ${ }^{14}$ - qui a permis à Goldmann de ne sombrer ni dans ce qu'il appelle le pessimisme radical de la dialectique négative ni dans un utopisme optimiste attribué à Ernst Bloch.

\section{Bibliographie}

Adorno, T. W. et Goldmann, L. (1973). Dialogue. ctes du Deuxième colloque international sur la sociologie de la littérature (Royaumont, 12-14 janvier 1968). Première publication dans la Revue de I'Institut de Sociologie, nos 3-4 (dossier "Hommage à Lucien Goldmann »), 1973, pp. 525-542. Date de consultation 3.09.2015. http://ressources-socius.info/index.php/reeditions/18-reeditions-darticles/134-dialogue

Aydın, U. U. (2008). Sihir ve Ütopya. Tolkien'in Yüzüklerin Efendisi'nde Romantik Eleştiri [Magie et utopie. La critique romantique dans le Seigneur des anneaux de Tolkien]. Istanbul : Versus.

Cahiers de Royaumont (1965). Le Concept d'Information dans la Science Contemporaine. Paris : Editions de Minuit. Date de consultation 12.11.215. https:// archive.org/stream/concept-information/Le\%20Concept\%20d'Information \%20 dans\%20la\%20Science\%20Contemporaine_djvu.txt

Cohen, M. (1944). The Wager of Lucien Goldmann : tragedy, dialectics, and a hidden god. New Jersey: Princeton University Press.

Dervilles, G. (1997). Le Pouvoir des médias. Mythes et réalités. Grenoble : Presses Universitaires de Grenoble.

Goldmann, L., et alii. (1966). Kierkegaard vivant. Paris : Gallimard.

Goldmann, L., et alii. (1970). Structuralisme et marxisme. Paris : 10/18.

13 La question du pari (tragique ou dialectique) est désignée ainsi chez Goldmann : "Le pari est fondamentalement l'expression du paradoxe de l'homme et de sa condition. Pour que l'homme vive en tant qu'homme, il doit engager sa vie sans réserve, dans l'espoir d'une valeur authentique dont le signe le plus clair est qu'elle est réalité » (1980, p. 187). Voir aussi Michael Löwy, "Lucien Goldmann, marxiste pascalien ", http://www.europe-solidaire.org/spip.php?article21214 [04.08.2015]

14 "Risque, possibilité d'échec, espoir de réussite et ce qui est la synthèse des trois, une foi qui est pari, voilà les éléments constitutifs de la condition humaine" (1976, p. 337). 
Goldmann, L. (1946). La communauté humaine et l'univers chez Kant. Paris : P.U.F.

Goldmann, L. (1970). Marxisme et sciences humaines. Paris : Gallimard.

Goldmann, L. (1973). Lukacs et Heidegger. Paris: Denoël/Gonthier.

Goldmann, L. (1975). La création culturelle dans la société moderne. Paris: Denoël/Gonthier (1ére édition 1971).

Goldmann, L. (1976). Le Dieu caché. Paris : Gallimard.

Goldmann, L. (1978a). Sciences humaines et philosophie politique. Paris : Denoël/Gonthier.

Goldmann, L. (1978b). Epistémologie et philosophie politique. Paris : Denoël/ Gonthier.

Goldmann, L. (1980). Recherches dialectiques. Paris : Gallimard.

Goldmann, L. (2001). Introduction aux premiers écrits de Georges Lukacs. Lukacs, in La théorie du roman (pp. 156-190). Paris : Gallimard.

Hall, S. (1997). Codage/Décodage. Sociologie de la communication, volume $1, \mathrm{n}^{\circ} 1$, p. 59-71. Date de consultation 17.11.2015. http://www.persee.fr/doc/ reso_004357302_1997_mon_1_1_3832

Jay, M. (1986). Lukacs, Bloch et la lutte pour un concept marxiste de totalité. Réification et Utopie. Ernst Bloch \& György Lukacs un siècle après. Actes du colloque Goethe Paris 1985. Paris: Editions Actes Sud.

Löwy, M. et Sayre, R. (1992). Révolte et mélancolie. Le romantisme à contrecourant de la modernité. Paris: Payot.

Löwy, M. (1985). Paysages de la vérité. Introduction à une sociologie critique de la connaissance. Paris: Editions Anthropos.

Löwy, M (2009). Lucien Goldmann, marxiste pascalien. Date de consultation 04.08.2015. http://www.europe-solidaire.org/spip.php?article21214

Lukacs, G. (2001). Histoire et conscience de classe. Paris : Editions de Minuit.

Maigret, E. (2007). Sociologie de la communication et des médias. Paris : Armand Colin.

Mattelart, A. et Neveu, E. (2008). Introduction aux cultural studies. Paris : La Découverte.

Naïr, S. et Löwy, M. (1973). Lucien Goldmann ou la dialectique de la totalité. Paris : Editions Seghers.

Palmier, J-M. (1974). Goldmann vivant. Esthétique et marxisme. Paris : 10/18, p.107-131.

Zima, P. V. (1973). Goldmann. Dialectique de l'immanence. Paris : PsychotèqueEditions Universitaires. 